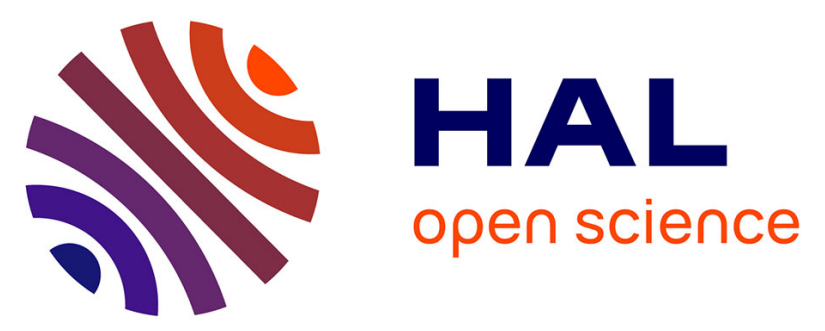

\title{
NEURAL-NETWORK METAMODELLING FOR THE PREDICTION OF THE PRESSURE DROP OF A FLUID PASSING THROUGH METALLIC POROUS MEDIUM
}

Eddy El Tabach, Nicolas Gascoin, Philippe Gillard

\section{To cite this version:}

Eddy El Tabach, Nicolas Gascoin, Philippe Gillard. NEURAL-NETWORK METAMODELLING FOR THE PREDICTION OF THE PRESSURE DROP OF A FLUID PASSING THROUGH METALLIC POROUS MEDIUM. Journal of Porous Media, 2014, 10.1615/JPorMedia.v17.i5.50 . hal-01253267

\section{HAL Id: hal-01253267 https://hal.science/hal-01253267}

Submitted on 9 Jan 2016

HAL is a multi-disciplinary open access archive for the deposit and dissemination of scientific research documents, whether they are published or not. The documents may come from teaching and research institutions in France or abroad, or from public or private research centers.
L'archive ouverte pluridisciplinaire HAL, est destinée au dépôt et à la diffusion de documents scientifiques de niveau recherche, publiés ou non, émanant des établissements d'enseignement et de recherche français ou étrangers, des laboratoires publics ou privés. 


\title{
Neural-network metamodelling for the prediction of the pressure drop of a fluid passing through metallic porous medium
}

\author{
Eddy El Tabach*, Nicolas Gascoin, \& Philippe Gillard \\ PRISME laboratory, University of Orleans, 63 avenue de Lattre de Tassigny, 18000 Bourges, \\ France.
}

*Address all correspondence to Eddy El Tabach E-mail: eddy.el-tabach@univ-orleans.fr

\begin{abstract}
The pressure drop across metallic porous medium is a critical element in cooling aerospace engineering application. This paper presents a metamodel based on artificial neural networks (ANN) for estimating the pressure drop through metallic porous media. The ANN is developed using experimental data obtained from an experimental bench, developed at PRISME laboratory, which ensure the monitoring of temperature, pressure and mass flow rate in stationary and transient conditions. For each case the gas pressure which crosses the metallic porous material is measured as a function of inlet gas pressure, gas mass flow rate and temperature. The optimal feedforward ANN architecture with error backpropagation (BPNN) was determined by the cross validation method. The ANN architecture having 35 hidden neurons gives the best choice. Comparing the modelled values by ANN with the experimental data indicates that neural network model provide accurate results. The performance of the ANN model is compared with a metamodelling method using multilinear regression approximation.
\end{abstract}


KEY WORDS: Simulation metamodelling, Artificial neural networks, Backpropagation, Permeation, Porous medium, SCRamjet cooling, numerical simulation

\begin{tabular}{|c|c|c|c|}
\hline \multicolumn{4}{|c|}{ NOMENCLATURE } \\
\hline$m$ & number of hidden nodes & $T$ & dimensional temperature $(\mathrm{K})$ \\
\hline$n$ & number of input nodes & $t$ & target value \\
\hline$O_{i}$ & $\begin{array}{l}\text { simulation output measures, } \\
\text { corresponding to } X_{i}\end{array}$ & $W_{i j}$ & $\begin{array}{l}\text { connection weights between the } \\
\text { input layer and the hidden layer }\end{array}$ \\
\hline$P$ & gas pressure $(\mathrm{Pa})$ & $W_{j k}$ & $\begin{array}{l}\text { connection weights between the } \\
\text { hidden layer and the output layer }\end{array}$ \\
\hline$p$ & number of output nodes & $X_{i}$ & $\begin{array}{l}\text { particular input parameter } \\
\text { setting, } i\end{array}$ \\
\hline$q$ & gas mass flow rate $\left(\mathrm{kg} \mathrm{s}^{-1}\right)$ & Sul & ripts \\
\hline$S$ & transfer function & in & inlet \\
\hline$s$ & number of patterns & out & outlet \\
\hline
\end{tabular}

\section{INTRODUCTION}

One of the many issues encountered in the development of future hypersonic vehicles is the problem of protecting the engines and airframe from high heat loads encountered at hypersonic speeds. At flight speeds near Mach 4, the air taken on board these vehicles will be too hot to cool the engines and airframe. Therefore, it will be necessary to study and develop other light weight and high-temperature materials. Among the materials which are intended to be used for high-speed flight are the metallic and the composite ones. The materials and cooling techniques and their interaction with aero-thermal loads must be addressed.

In the literature, we can found different studies in relationship with this need, experimentally (Langener et al., 2011; Gascoin et al., 2012) or numerically (Jaber and Ziad Saghir, 2011; Shahnazari and Vahabikashi, 2011); even mathematically (Kim and Park, 1999; Martin and Boyd, 2008). Such studies are not only dedicated to the flow description but also to the heat transfers (Kim and Park, 1999; Krishna et al., 2008; Younis, 2010); for 
example for configurations close to fuel cooled structures (Zhao and Chen, 2003) and also for geophysics applications (Hadim, 1994). The flows in porous media are widely studied under common operating conditions.

A lot of studies, often under high pressure (up to 2MPa) are available for ambient conditions or average temperature conditions (under 800K) (Park and Lawrence, 2003; Langener, 2011) or for low pressure and high temperatures (Kuhn and Hald, 2008). But only few are dedicated to both high temperature and high pressure conditions. Numerous equations (derived from Brinkman's equation) which relate pressure drop $\left(\triangle P=P_{\text {in }}-P_{\text {out }}\right)$ through the porous media to the gas velocity have been published (Martin and Boyd, 2008; Khan and Reddy Gorla, 2010; Gascoin et al., 2012). They are based on coefficient, whose physical meaning is not evident. Furthermore, the dynamic viscosity, which appears in these equations, is often considered to be that of the fluid. It remains constant in most of experimental and numerical works. Nevertheless, this is questionable because this parameter varies across the porous medium in the case of reactive flow or a non-uniform temperature system.

In this paper, we have used a simulation metamodelling to better simulate the pressure drop of nitrogen passing through the porous media (Stainless Steel) by taking into account both high temperature and high pressure conditions. Simulation metamodelling is based on the substitution of the complex simulation model by an approximation of the input-output relationship. Metamodelling, first proposed by Blanning (1975), makes the computations much faster, allowing for more cases to be studied. This wider exploration of the input variables improves the understanding of the model and it permits to carry out otherwise time consuming sensitivity analysis or solution optimization (Broad, 2004, Kleijnen et al., 2010), at the cost however of a lower accuracy of the outputs. Originally based on regression methods (Cohen et al., 2003; Freedman, 2008), metamodels now use various approaches such 
as artificial neural networks (ANN) or kriging (Kleijnen, 2009; Ankenman et al., 2010). ANN has the advantage over regression that the form of the model needs not to be pre-determined. Moreover, ANN can theoretically approximate any function to any level of accuracy (El Tabach et al., 2007), which is very interesting when the governing physical mechanisms are highly non-linear like in high velocity fluid flow in porous medium. The database was built with three input parameters (inlet gas mass flow rate, inlet gas pressure and the temperature) and the outlet gas pressure as output parameters, using the results coming from an experimental bench. An artificial neural network has been trained and tested on this database using the error backpropagation algorithm and cross validation.

\section{THE EXPERIMENTAL BENCH AND SPECIFIC SET-UP}

The COMPARER pyrolysis test bench is used to pressurize and to heat the fuel under flow conditions (Gascoin et al., 2011). Its main characteristics are the following:

- Maximum operating conditions: $1800 \mathrm{~K}, 8 \mathrm{MPa}, 0.0006 \mathrm{~kg} \cdot \mathrm{s}^{-1}$ for liquid fluid and $0.006 \mathrm{~kg} . \mathrm{s}^{-1}$ for gas.

- Sensors: 5 mass flow rates, 5 pressure transducers, over 10 K-type and R-type thermocouples with data acquisition system (16 bits, 48 channels, $0.1 \mathrm{~Hz}$ ).

A permeation test cell contains the porous sample (FIG. 1). This cell is inserted inside the furnace of the COMPARER bench and it is connected to the fluid supply system and to the appropriate sensors. The permeable medium bounds the cell in two high and low pressure chambers (upstream and downstream to the porous medium respectively). These chambers are noted HPC and LPC in this paper. An inlet pipe provides the fuel into the system. This cell is connected to a dynamic sampling system to get hot pressurized samples at three location points in the cell. Despite its small size (external diameter of 40mm), it enables measuring the temperature, pressure and mass flow rate on each side of the porous sample. 
The permeable medium can be changed. In the present work, an isotropic stainless steel material is preferred to composite one to avoid considering complex microstructure (fibres, layers). It is characterized by a porosity around $30 \%$, a grain diameter of $14.1 \mu \mathrm{m}$ and a pore diameter of $4.1 \mu \mathrm{m}$ (Gascoin et al., 2012). Further geometrical information can be found in Gascoin et al. (2012).

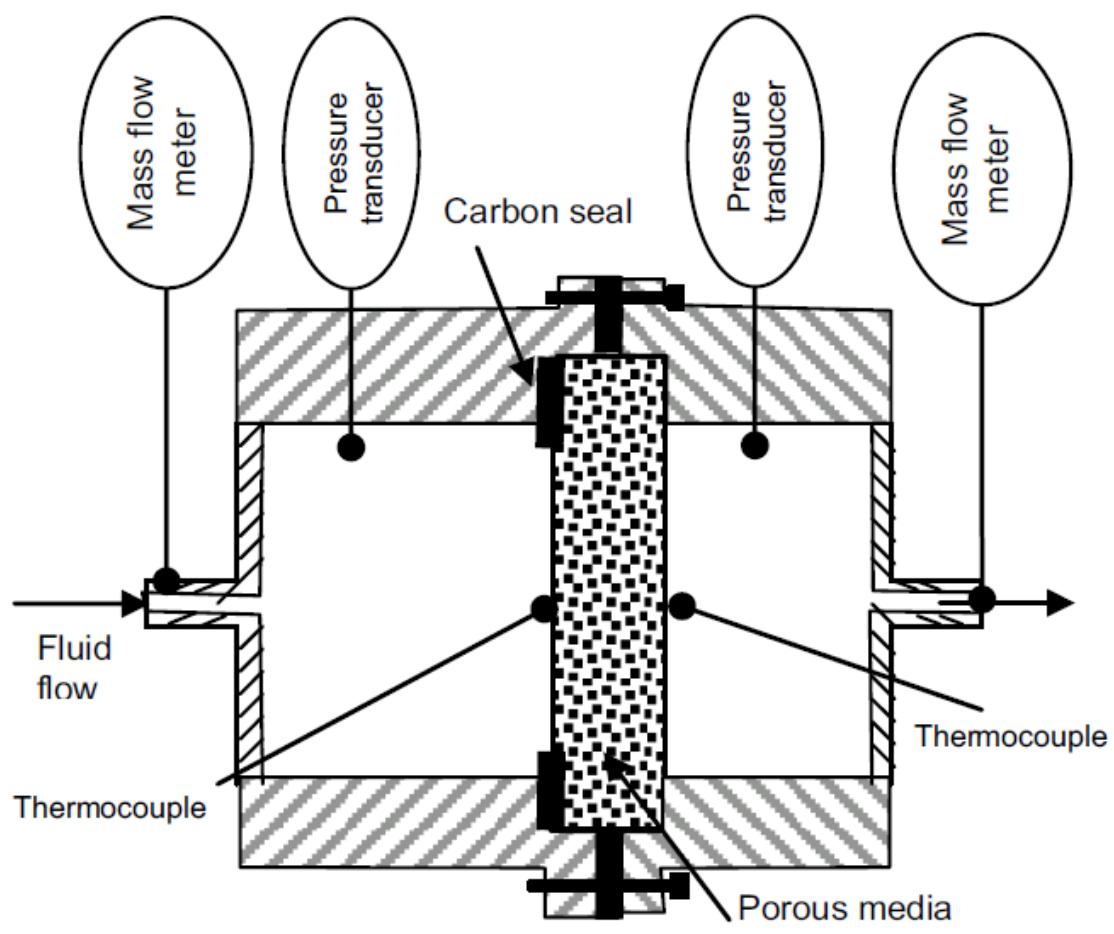

FIG. 1: Schematic of the permeation test cell with porous medium and associated measures (Gascoin, 2011).

The different test conditions and most of the possible combinations have been achieved:

- Temperature set-up: from $300 \mathrm{~K}$ to $1200 \mathrm{~K}$ by step of $100 \mathrm{~K}$.

- Absolute pressure: from $0.05 \mathrm{MPa}$ (depressurized system) to 6MPa. 
- Two experimental protocols have been tested: one by measuring the mass flow rate and changing the upstream pressure and a second by measuring the pressure drop and changing the upstream mass flow rate.

\section{CONSTRUCTION OF THE ANN METAMODEL}

\subsection{Artificial neural networks}

The metamodel adopted in this paper is based upon the use of artificial neural networks (ANN). ANN operate like a "black box" model, requiring no detailed information about the physical parameters of the system. Instead, they learn the relationship between the input and output parameters as a result of training with previously recorded data. ANN can handle large and complex systems with many interrelated parameters.

\subsection{Construction of the database}

The database was built using experimental data which are obtained from the developed experimental bench with input parameters: gas inlet pressure $\left(P_{i n}\right)$, inlet gas mass flow rate $\left(q_{i n}\right)$ and temperature $(T)$ varying in a range of representative values: 35 values between $0.1 \mathrm{MPa}$ and $1.3 \mathrm{MPa}$ for $\mathrm{P}_{\mathrm{in}} ; 35$ values between $0.00008 \mathrm{~kg} / \mathrm{s}$ and $0.0011 \mathrm{~kg} / \mathrm{s}$ for $\mathrm{q}_{\text {in }}$ and 10 values between $290 \mathrm{~K}$ and $1174 \mathrm{~K}$ for T. In total; the database contains an appreciable size of 350 experimental tests.

The present database was subdivided in three subsets. A first subset (174 experimental tests) is used to train the networks. A second one (88 experimental tests) is used to test the ANN models to determine when to stop the training stage. The third subset (88 experimental tests) is used to validate the performance of the selected model on unseen cases. It should be 
noted that the training subset should contain the widest variety of patterns, since ANN are more reliable if they are used as an interpolation tool for generalization on new cases.

Note also that each input or output parameter has been normalized relative to its minimum and maximum values observed in the data, allowing for faster training by preventing larger numbers from overriding smaller ones. Normalization of an arbitrary parameter, $X$, can be carried out using Eq. 1 .

$$
X_{n o r m}=\frac{\left(X-X_{\min }\right)}{\left(X_{\max }-X_{\min }\right)}
$$

where $X_{n o r m}$ is the normalized value, and $X_{\max }$ and $X_{\min }$ are the maximum and minimum values of $X$.

\subsection{Construction of artificial neural network models}

A brief description of the model and program used in this study will be given in this section. For a detailed description, evaluation, and discussion on the overall performance of the model, readers are referred to Najjar et al. (1997).

ANN are composed of a set of elements of calculation (nodes) connected to each other. The most popular type of network is the multilayer backpropagation neural network (BPNN) which is used in the present study. The architecture of a typical 3-layer backpropagation neural network is shown in Fig. 2. Mathematically, a 3-layer ANN with $n, m$, and $p$ the number of input, hidden and output nodes respectively, is based on the following equation:

$$
O_{k}=S\left(\sum_{j=1}^{m} W_{j k} \times S\left(\sum_{i=1}^{n} W_{i j} X_{i}\right)\right)
$$


where $O_{k}$ are the output values and $X_{i}$ the input values of the network; $W_{i j}$, the connection weights between the input layer and the hidden layer; $W_{j k}$, the connection weights between the hidden layer and the output layer; $S$ is a transfer function. The sigmoidal function (Eq. 3) was used in the present study.

$$
S(x)=\frac{1}{1+e^{-x}}
$$

The input from each node in the previous layer $\left(X_{i}\right)$ is multiplied by an adjustable connection weight $\left(W_{i j}\right)$. At each node, the weighted input signals are summed and an adjustable threshold value $\left(W_{j}\right)$ is added. This combined input $A_{j}$ is then passed through the non-linear transfer function $\mathrm{S}$ to produce the output of the node $\left(O_{j}\right)$. The output of one node contributes to the input to the nodes in the next layer. This process is illustrated in Fig. 2. In the present study, all connection weights $\left(W_{i j}\right)$ and the thresholds $\left(W_{j}\right)$ are initialized to random values in the range of [+1, -1] (Najjar et al., 1997).

The learning process of BPNN is based on a series of connection weight adjustments in order to minimize a global error between predicted outputs and target values. It relies on a search technique (e.g. gradient descent) of the connecting weights yielding a minimum error. Inputs are first propagated forward through each layer of the network. Errors between outputs and target values are then propagated backwards and the connection weights are modified according to a specific learning algorithm (delta rule) to reduce the overall error. This forward-backward process is carried out for each epoch (set of training patterns used to compute the global error), and is repeated until predicted outputs and target answers coincide within a given tolerance.

The most common convergence criterion is the average squared error (ASE) defined as: 


$$
A S E=\frac{1}{p} \times \frac{1}{s} \times \sum_{q=1}^{s} \sum_{k=1}^{p}\left(t_{q k}-O_{q k}\right)^{2}
$$

where $O_{q k}$ ant $t_{q k}$ are respectively the predicted and target value of the output node k for the pattern $q, s$ is the number of patterns, and $p$ is the number of output nodes. It should be noted that any level of agreement between predicted and target vectors can be achieved by providing a sufficient number of training cycles to be carried out. Such an overtraining is however detrimental to the capacity of the network to generalize from unseen data (a network that can accurately predict the output of the testing patterns is said to have generalized). It is thus preferable to compute the ASE both on training and testing patterns during training cycles for optimum convergence: this process is called cross-validation (FIG. 3).

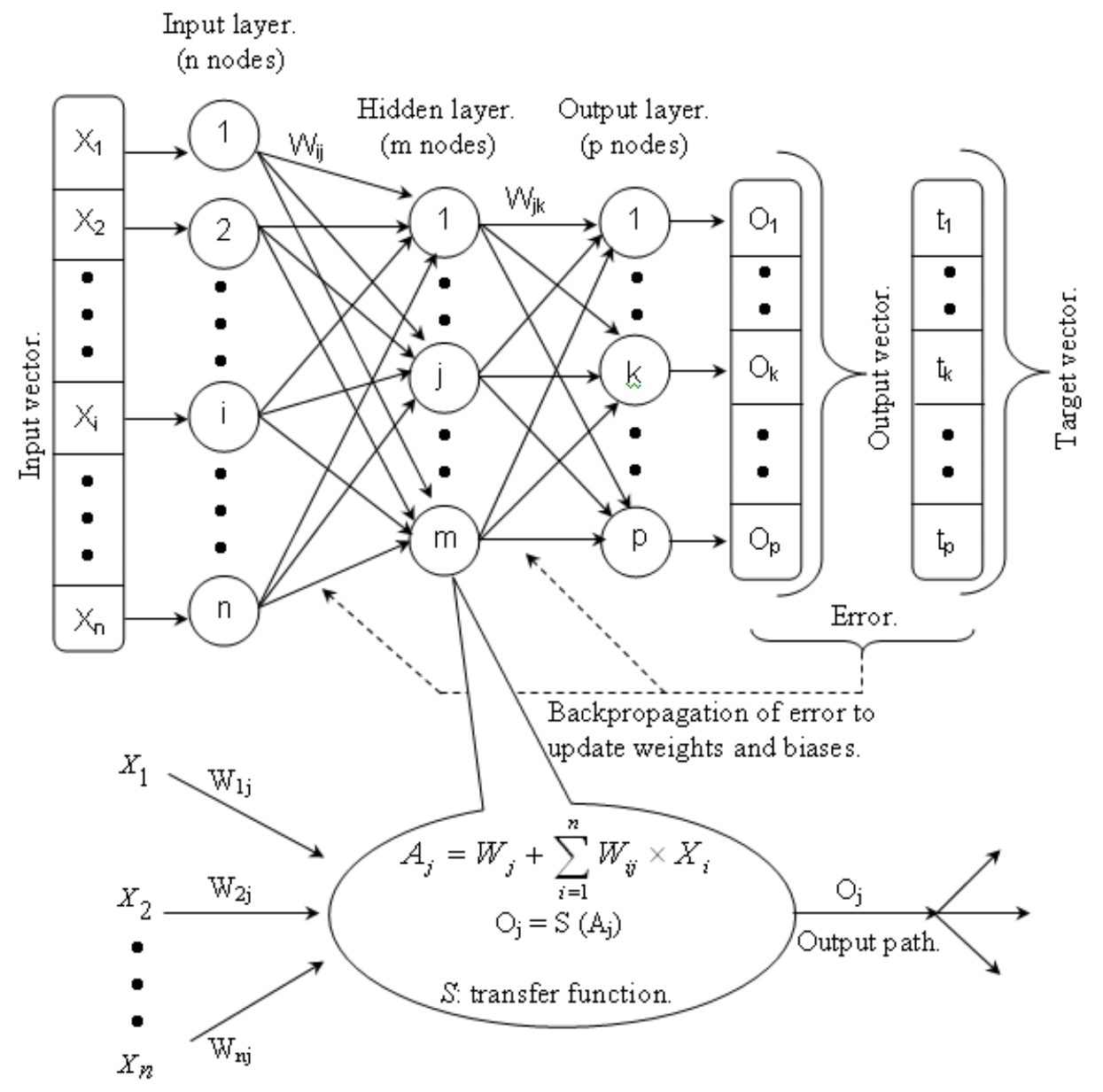

FIG. 2: Architecture of a typical multilayer backpropagation artificial neural network 


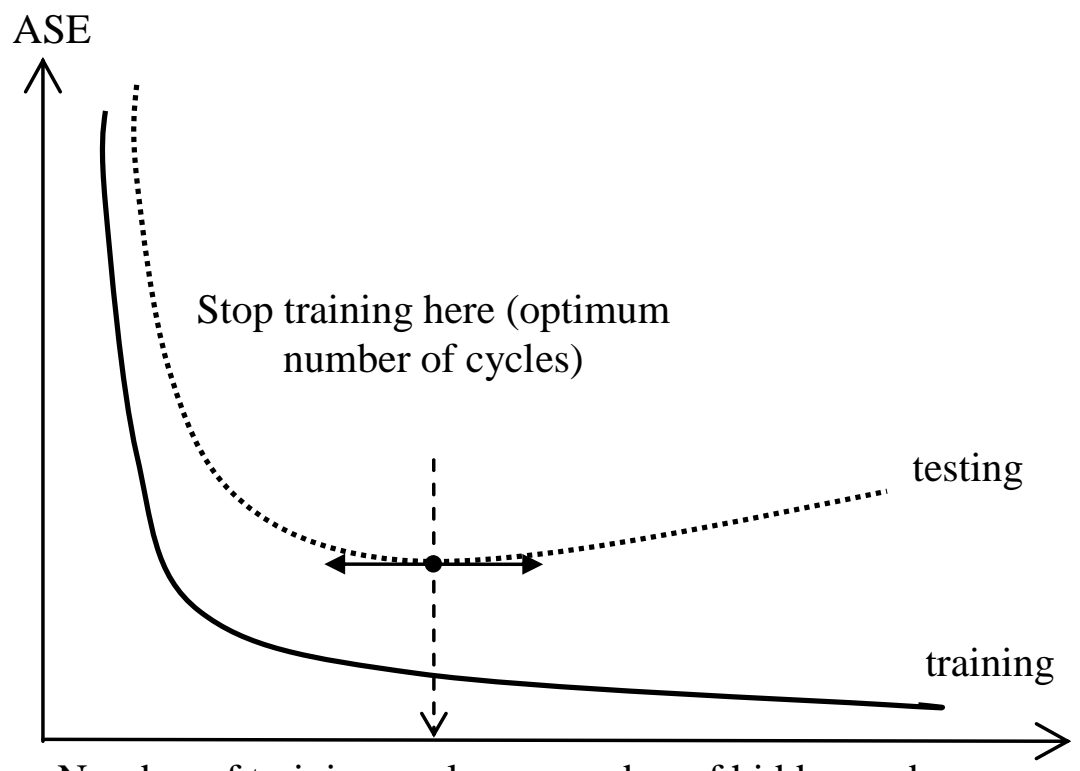

Number of training cycles or number of hidden nodes

FIG. 3: Convergence criterion and optimum network architecture.

\subsection{Optimum network architecture}

The overall performance of an ANN is very dependent on the number of hidden layers and of hidden nodes in each hidden layer. In the usual case of a 3-layer BPNN (one hidden layer), the optimum number of hidden nodes can be assessed by cross-validation in the same way as the optimum number of training cycles (Fig. 3). Starting with a few hidden nodes, more nodes are added up as the ASE computed on the testing patterns decreases. The number of hidden nodes beyond which the error starts to increase again is taken as the optimum.

In the present study, a neural network relating inputs $\left\{X_{1}, X_{2}, \ldots, X_{n}\right\}$ to outputs $\left\{O_{1}, O_{2}\right.$, $\left.\ldots, O_{p}\right\}$ and containing one hidden layer with $m$ hidden nodes will be noted:

$$
\left\{O_{1}, O_{2}, \ldots, O_{p}\right\}=\mathrm{ANN}_{\text {n棡焵 }}\left\{X_{1}, X_{2}, \ldots, X_{n}\right\}
$$

In our case, the outlet gas pressure $\left(P_{\text {out }}\right)$ is sought as a function of $P_{i n}, q_{i n}$ and $T$. So, it is possible to compute $P_{\text {out }}$ by using a BPNN model with one node in the output layer (Eq. 6). It could be noticed that usually the pressure drop through the porous medium is investigated as 
a function of the through flow rate. In this present study, the choice of the outlet pressure is preferred to limit the impact of experimental uncertainties due to the two pressure transducers (which are generally multiplied if compared to a single transducer). In addition, since the upstream pressure is an inlet parameter, looking at the pressure drop or at the pressure outlet is equivalent when focusing on the behaviour of the ANN model. As can be observed in FIG. 4 the optimal value of ASE was calculated while using 35 nodes in the hidden layer for our model.

$$
\left\{P_{\text {out }}\right\}=\mathrm{ANN}_{3-35-1}\left\{P_{i n}, q_{\text {in }}, T\right\}
$$

The ASE values for the training, testing and validation phases for the optimal artificial neural network model $\left(\mathrm{ANN}_{3-35-1}\right)$ are respectively $0.15 \times 10^{-4}, 1.01 \times 10^{-4}$ and $1.69 \times 10^{-4}$. Somewhat larger value of the ASE is logically obtained during the validation phase.

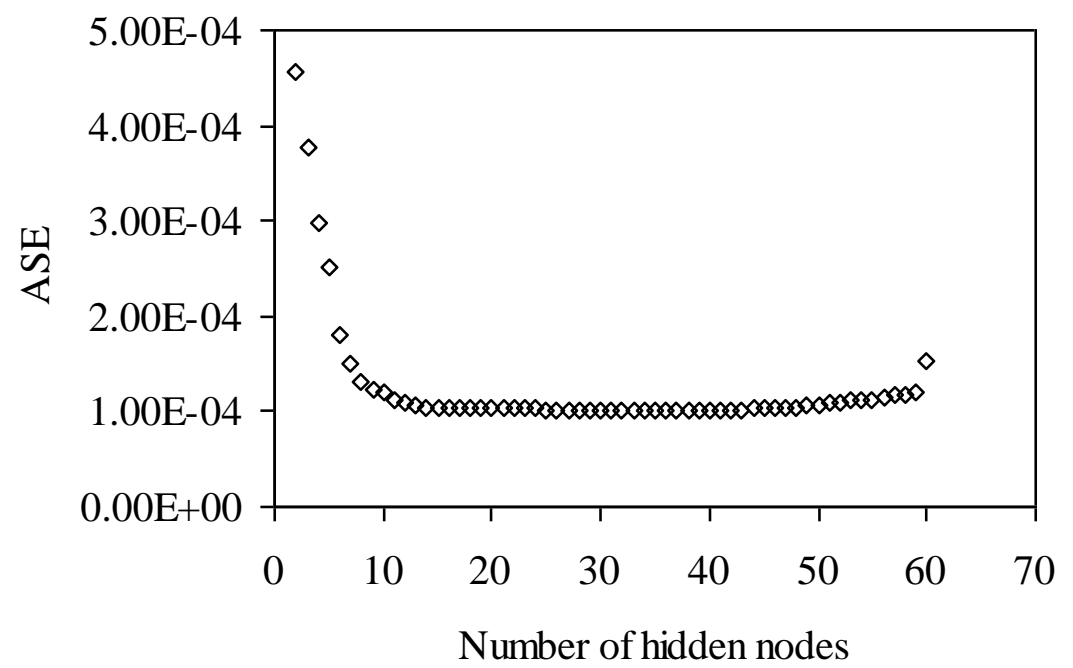

FIG. 4: Absolute squared error (ASE) variations with the number of hidden nodes for the testing data subset. 


\subsection{Discussion of the performance of the model}

The performance of the model is assessed by comparing target and predicted values $Y_{i}$ and $\hat{Y}_{i}$. If predicted values are plotted against target values, the distance of these points to the bisectrix gives an indication about how close the model fits the data. FIG. 5 shows the comparison between the BPNN predicted values and the target values for $P_{\text {out }}$ on previously unseen data (validation phase). On the same graphs the best fit line through the origin is also plotted and the coefficient of determination $\mathrm{R}^{2}$ for this line is computed according to Eq. 7:

$$
R^{2}=1-\frac{\frac{1}{N} \sum_{i=1}^{N}\left(Y_{i}-\hat{Y}_{i}\right)^{2}}{\frac{1}{N-1} \sum_{i=1}^{N}\left(Y_{i}-\bar{Y}_{i}\right)^{2}}
$$

where $N$ is the number of data, $\hat{Y}_{i}$ is the value predicted by the model, $Y_{i}$ is the target value and $\overline{Y_{i}}$ is the mean of the $N$ target values. $R^{2}$ coefficients close to unity indicate a high degree of linearity between predicted and target values. Associated with a best fit line slope close to unity, it indicates a high model prediction accuracy.

A basis of comparison for BPNN performance is usually sought in multiple linear regression (Tabachnick, 2007), a more ubiquitous prediction tool in fluid flow through porous media research. Least square parameter fitting for a linear model expressing $P_{\text {out }}$ as a function of $P_{i n}, q_{i n}$, and $T$ (model 2) is performed on the same training database subset as for BPNN model. This model is tested to predict the never-seen data from the BPNN validation database subset. A greater scatter, quantified by a smaller $R^{2}$ value, is obtained for multiple linear regression, as compared to backpropagation neural networks. It is also noted that the

trend line deviates somewhat from the 1:1 line in the case of model 2. The coefficients of determination for model 1 and model 2 for all training, testing and validation phases are 
given in Table 1. The multiple linear regression model fails to predict the variations of $P_{\text {out }}$. The poor performance of the linear model is not surprising: the physical phenomena captured in the database are quite complex and highly non linear. In artificial neural networks nonlinearity is accounted for by the use of transfer functions (Eq. 3), while complexity can be controlled by varying the number of hidden nodes. Artificial neural networks appears as a powerful prediction tool in the present case. 

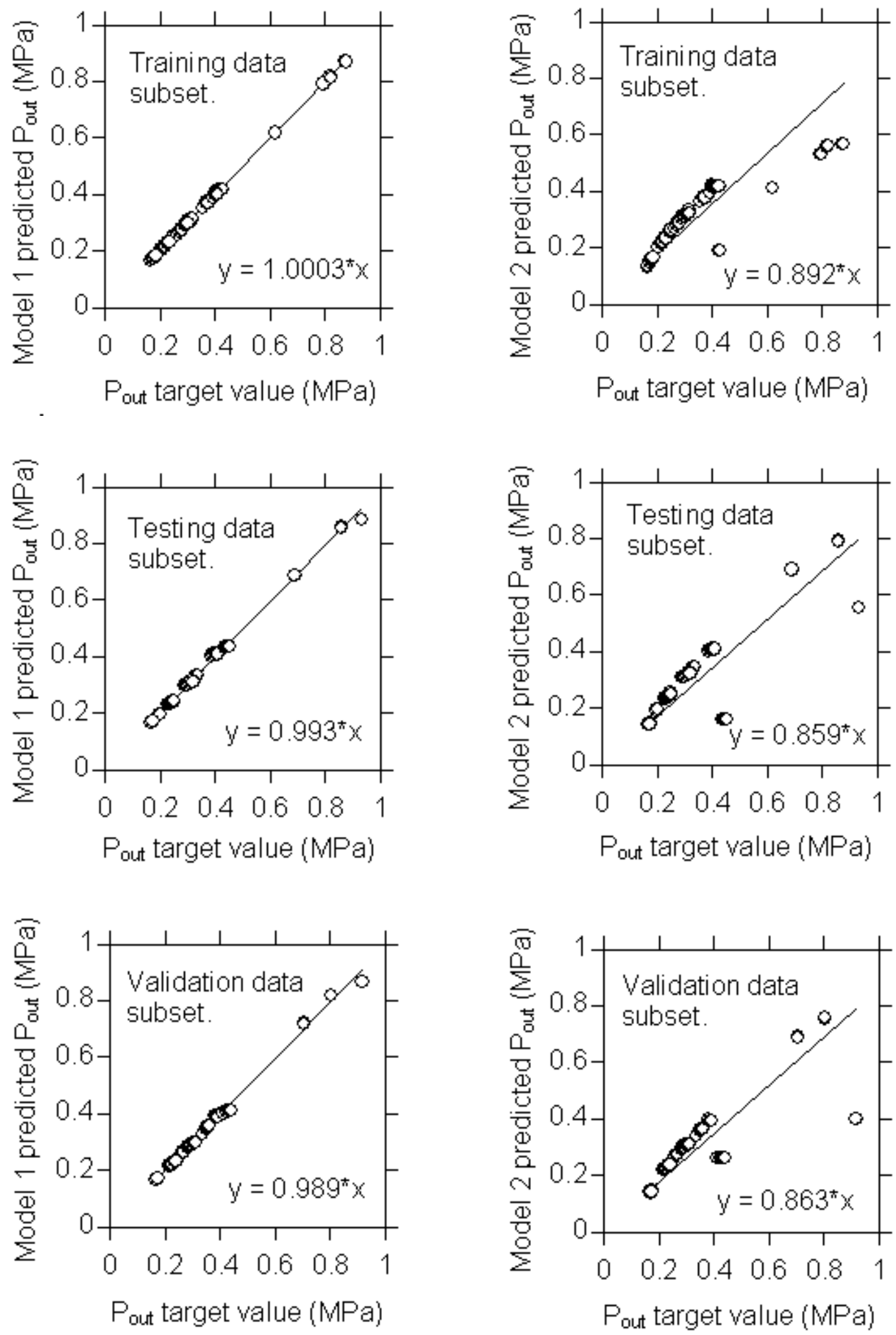

FIG. 5: Comparison between target and predicted values for $P_{\text {out }}$ using BPNN (model 1) and multi-linear regression (model 2) for all data subset. 
Table 1: $R^{2}$ values between target and predicted outputs for all models

\begin{tabular}{lll}
\hline$R^{2}$ & Neural network (model 1) & Multiple linear regression (model 2) \\
\hline Training phase & 0.999 & 0.682 \\
Testing phase & 0.997 & 0.639 \\
Validation phase & 0.996 & 0.592 \\
\hline
\end{tabular}

The evolution of the predicted outlet pressure $\left(P_{\text {out }}\right)$ using BPNN as a function of temperature inside the porous medium corresponds to a decrease when the temperature increases (FIG. 7). The density decreases due to the thermal rise and since the mass flow rate inside the medium is kept constant, the mean fluid velocity increases. Thus, the outlet pressure decreases; which means that the pressure drop increases (for a given curve corresponding to a fixed inlet pressure). This is clearly understandable when paying attention to the Brinkman equation. It is thus very important to note that the ANN approach is able to reproduce physical variations.

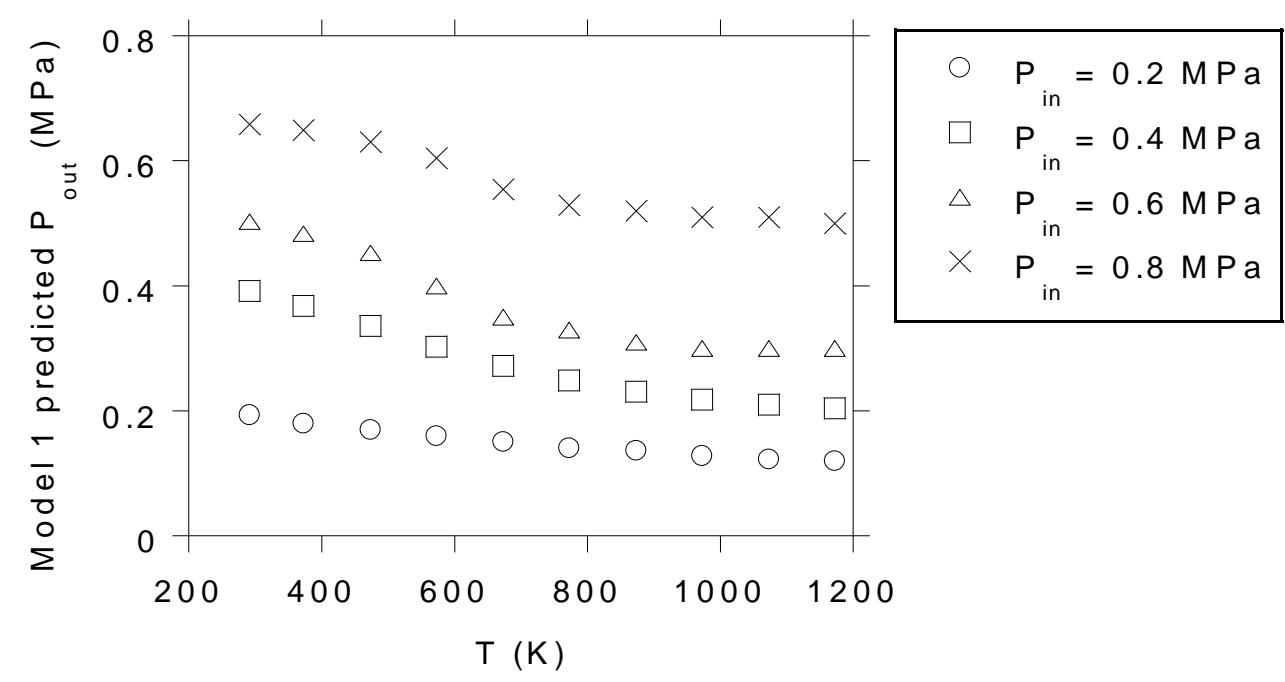

FIG.7: Model 1 predicted $\mathrm{P}_{\text {out }}$ variations with the temperature for $\mathrm{q}_{\text {in }}=1 \mathrm{~g} / \mathrm{s}$. 
In addition, it can be seen that the same pressure drop $\left(\Delta P=P_{i n}-P_{\text {out }}\right)$ is found for several couples of temperature/inlet pressure (FIG. 8). For example a pressure drop of $0.2 \mathrm{MPa}$ is reached for $\left(\mathrm{P}_{\mathrm{in}}=0.4 \mathrm{MPa}\right.$ and $\left.\mathrm{T}=1173.15 \mathrm{~K}\right)$ and for $\left(\mathrm{P}_{\mathrm{in}}=0.6 \mathrm{MPa}\right.$ and $\left.\mathrm{T}=573.15 \mathrm{~K}\right)$. This means that the pressure is divided by a factor 2 at high temperature while for about half of this temperature, the pressure losses only $33 \%$. To divide the inlet pressure of $0.6 \mathrm{MPa}$ by a factor 2 , the temperature should be about $873.15 \mathrm{~K}$. As a consequence, it can be concluded physically, that the temperature has a higher effect than the pressure itself. The thermal effect much increases the fluid velocity within the porous media (more than what the pressure does). This is the thermodynamic consequence of pressure and temperature parameters on the fluid density. This could be a way to get information of phenomena within the porous media where no direct measure seems to be possible for the fluid properties.

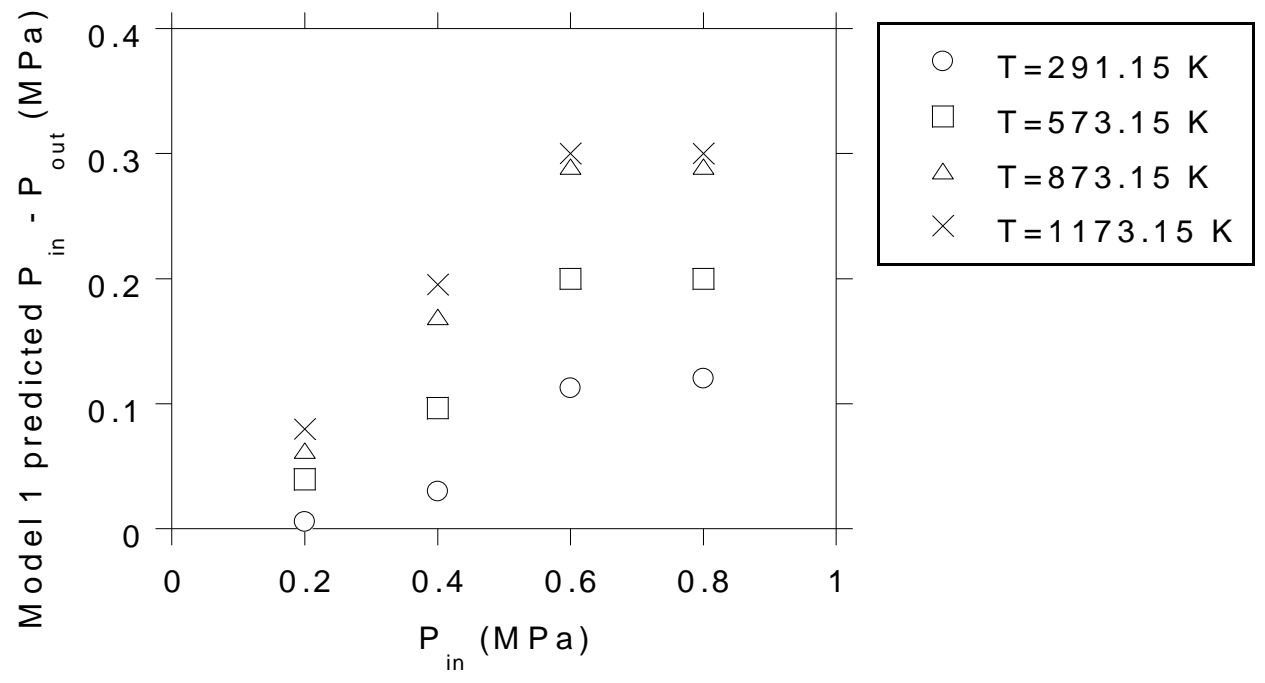

FIG. 8: Model 1 predicted $\Delta P$ variations with $\mathrm{P}_{\text {in }}$ for $\mathrm{q}_{\text {in }}=1 \mathrm{~g} / \mathrm{s}$.

\section{CONCLUSIONS}

A metamodel based on artificial neural networks has been used to predict the nitrogen pressure which crossed a metallic porous media (Stainless steel). Based on experimental data, 
the optimum architecture of artificial neural network was trained and validated, in order to generalise the prediction of gas pressure which crossed the studied porous media to cases not included in the database. The validation showed excellent performance of this metamodel for the prediction of gas transfer in the porous media.

This study is a contribution to the growing evidence of the benefits of simulation metamodels in Aerospace engineering. This important result may be applied to automate $P_{\text {out }}$ estimations which utilized in space flight applications without prior knowledge of correct material parameters.

\section{ACKNOWLEGEMENTS}

The authors would like to sincerely thank Fau G. from PRISME and Blanc D. from IUT Bourges for their technical and scientific support involving the computations and the experiments.

\section{REFERENCES}

Ankenman, B., Nelson, B. L., and Staum J., Stochastic kriging for simulation metamodeling. Operations Research, vol. 58, no. 2, pp. 371-382, 2010.

Blanning, R.W., The construction and implementation of metamodels, Simulation, vol. 24, no. 6 , pp. $177-184,1975$.

Broad, D.R., Dandy, G.C., and Maier, H.R., A metamodelling approach to water distribution system optimisation. World Water and Environmental Resources Congress, Salt Lake City, USA, June 27-July 1, 2004. 
Cohen, J., Cohen, P., West, S.G., and Aiken, L.S., Applied Multiple Regression/Correlation Analysis for the Behavioral Sciences, (3 ${ }^{\text {rd }}$ ed.), Hillsdale, NJ: Lawrence Erlbaum Associates, 2003.

El Tabach, E., Lancelot, L., Shahrour, I., and Najjar, Y., Use of artificial neural network simulation metamodelling to assess groundwater contamination in a road project, Mathematical and Computer Modelling, vol. 45, pp. 766-776, 2007.

Friedman, L.W., The simulation metamodel, Kluwer Academic Publishers, Norwell, MA, 1996.

Gascoin, N., Fau, G., Gillard, P., Kuhn, M., Bouchez, M., and Steelant, J., Comparaison of two permeation test benches and two determination methods for Darcy's and Forchheimer's permeabilities, J. Porous Media, vol. 15, no. 8, pp. 705-720, 2012.

Gascoin, N., High temperature and pressure reactive flows through porous media, International Journal of Multiphase Flow, vol. 37, pp. 24-35, 2011.

Hadim, A., Numerical Study of Non-Darcy Mixed Convection in a Vertical Porous Channel, Journal of thermophysics and heat transfer, vol. 8, no. 2, pp. 371-373, 1994.

Jaber, T.J. and Ziad Saghir, M., Three-dimensional study of permeability effect on convection in heterogeneous porous medium filled with a ternary hydrocarbon mixture, $\mathrm{J}$. Porous Media, vol. 14, no. 4, pp. 305-315, 2011.

Khan, W. A. and Reddy Gorla, R. S., Second law analysis for mixed convection in nonNewtonian fluids over a horizontal plate embedded in a porous medium, Special Topics Rev. Porous Media, vol. 1, no. 4, pp. 353-359, 2010. 
Kim, M.-Y. and Park, E.-J., Fully Discrete Mixed Finite Element Approximations for NonDarcy Flows in Porous Media, Computers and Mathematics with Applications vol. 38, pp. 113-129, 1999.

Kleijnen, J.P.C., Kriging metamodeling in simulation: A review. European Journal of Operational Research, vol. 192, pp. 707-716, 2009.

Kleijnen, J.P.C., van Beers, W., and van Nieuwenhuyse, I., Constrained optimization in expensive simulation: Novel approach. European Journal of Operational Research, vol. 202, no. 1, pp. 164-174, 2010.

Krishna, D.J., Basak, T., and Das, S.K., Natural convection in a heat generating hydrodynamically and thermally anisotropic non-Darcy porous medium, International Journal of Heat and Mass Transfer, vol. 51, pp. 4691-4703, 2008.

Kuhn, M. and Hald, H., Application of transpiration cooling for hot structures, RESPACE: Key Technologies for Reusable Space Systems, Note N. Fl. Mech. Mul. D., vol. 98, pp. 82103, 2008.

Langener, T., Wolfersdorf, J. von, and Steelant, J., Experimental investigations on transpiration cooling for scramjet applications using different coolants, AIAA Journal, vol. 49, no. 7, pp. 1409-1419, 2011.

Martin, A. and Boyd, I. D., Simulation of pyrolysis gas within a thermal protection system, 40th Thermophysics Conf., Seattle, USA, AIAA-3805, 2008.

Najjar, Y.M., Basheer, I.A., and Hajmeer, M.N., Computational neural networks for predictive microbiology: i. Methodology. International Journal of Food Microbiology vol. 34, pp. 27-49, 1997. 
Park, O.Y. and Lawrence, T.W., High Temperature Permeability of Carbon Cloth Phenolic Composite, $39^{\text {th }}$ AIAA/ASME/SAE/ASEE Joint Propulsion Conference and Exhibit 20-23, Huntsville, Alabama, AIAA 2003-5242, 2003.

Shahnazari, M.R. and Vahabikashi, A., Permeability prediction of porous media with variable porosity by investigation of Stokes flowover multi-particles, J. Porous Media, vol. 14, no. 3, pp. 243-250, 2011.

Tabachnick, B.G., and Fidell, L.S., Using multivariate statistics $\left(5^{\text {th }}\right.$ ed.), Boston: Allyn and Bacon, 2007.

Younis, L.B., Cross-flow heat exchanger embedded within a porous medium, J. Porous Media, vol. 13, no. 11, pp. 981-988, 2010.

Zhao J.Z. and Chen, T.S., Non-Darcy effects on nonparallel thermal instability of horizontal natural convection flow, Journal of Thermophysics and heat transfer, vol. 17, no. 2, AprilJune 2003. 